\title{
Application of Image Processing to Health Monitoring for Wire Rope of Lift systems
}

\author{
Keisuke Minagawa ${ }^{1}$ and Satoshi Fujita ${ }^{2}$ \\ ${ }^{1}$ Department of Mechanical Engineering, Saitama Institute of Technology, \\ 1690 Fusaiji, Fukaya, Saitama 369-0293, Japan, mina@sit.ac.jp \\ 2 Department of Mechanical Engineering, Tokyo Denki University, \\ 5 Senju-Asahi-cho, Adachi-ku, Tokyo 120-8551, Japan, sfujita@cck.dendai.ac.jp
}

Keywords: wire rope, diameter, red rust, monitoring, image processing.

\begin{abstract}
The rupture of wire ropes is one of the various severe accidents in lift systems. Before the rupture by aging degradation, the diameter of the wire ropes decreases and their surface is rusted. Thus diameters and red rust of wire ropes should be checked in periodic inspections of lift systems in Japan. The diameters are usually measured by using vernier calipers or scales, and red rust is checked by human inspection, so there are errors and differences among inspectors' judgements. Therefore, development of a new monitoring system for the diameters and red rust is required in order to ensure the quality of the inspection and to manage the inspection data efficiently. Meanwhile, image processing technology has recently been applied to various industries such as automatic driving vehicles. This paper proposes and constructs a health monitoring system for wire ropes using image processing. The system consists of a digital camera and a computer. The digital camera takes a photograph of a wire rope and the photograph is analysed by the computer. The diameter is calculated from the number of pixels of the rope, and red rust is detected by resolving the colour of the photograph into RGB data. This paper describes an image processing method for the inspection of wire ropes and the results of verification tests. The photographic conditions suitable for monitoring were investigated. As a result, the measurement error was less than $1 \%$ by adjusting photographic conditions.
\end{abstract}

\section{INTRODUCTION}

There are many kinds of wire ropes such as hoist rope, compensating rope and so on in a lift system. The hoist rope is an especially important component because it suspends a car, so the rupture of the rope causes severe accidents. In addition, roping of recent lifts has been complicated. For example, machine-room-less lifts have many sheaves compared to ordinary lifts in order to install the driving motor in the hoistway. Thus there are many bending points of ropes. The ropes may be damaged at these points. Therefore, maintaining rope safety is very important.

In 2011, a rupture accident of a hoist rope in a lift system occurred in Tokyo. According to the accident analysis report [1], the cause of the accident was aging degradation of the hoist rope and insufficiency of inspections. A decrease in the diameter of the rope and rust were confirmed in the rope, but detection of rupture was difficult because the rupture had started inside the rope. As a consequence of the accident, further investigations regarding safety measures of hoist ropes of lift systems and revisions of inspection methods were implemented. The investigation report described the relationship between the decrease in diameters of rope and the decrease in the strength of the rope [2]. In addition, wires in strands may break before the rupture of a rope. Therefore, the detection of wire breakage is also important.

In Japan, the diameter of the rope and red rust are checked during periodic inspections of lift systems. Usually the diameters are measured by using vernier calipers or scales, and red rust is checked with the eyes, so there are errors and differences among inspectors' evaluations. However, the inspection should be implemented homogenously and accurately from the viewpoint of the above-mentioned accidents. 
Image processing technology has recently been applied to various industries such as automatic driving vehicles, dynamic measurement [3] and so on. Computers and digital cameras are becoming smaller and have higher levels of performance. Thus, the application of image processing to various machines will increase in the future.

This paper proposes the application of image processing to monitor the health of wire ropes in lift systems in order to check the conditions of the ropes homogenously, accurately and speedily. The system proposed in this paper measures the diameters of the rope and detects red rust by using image processing. A photograph taken by a digital camera of a rope is used in this system, and the digital image is processed by using a computer. The diameter is calculated from the number of pixels of the rope, and red rust is detected by resolving the colour of the photograph into RGB data. A concept of the health monitoring system of wire rope using image processing, methodology used and test results for verification of the system are reported in this paper.

As mentioned above, the detection of wire breakage is also important. There is a possibility that wire breakage can be detected by image processing. However the system proposed in this paper only focuses on diameter measurement and red rust detection as a first step of rope monitoring by image processing.

Additionally, this paper is an extended version of the paper presented in the 7th Symposium on Lift and Escalator Technologies in 2017, whose title was "Health Monitoring System for Wire Rope Using Image Processing [4]”. Some content was added based on the discussion at the symposium.

\section{CONCEPT OF HEALTH MONITORING SYSTEM USING IMAGE PROCESSING}

The health monitoring system of wire ropes using image processing proposed in this paper is aimed at the inspection of the diameters and red rust of wire ropes. Figure 1 shows a concept diagram of the proposed system. The proposed system consists of a PC and a digital camera, and the camera is able to be set anyplace near the hoist rope, because the camera is small and is able to be connected with the PC by cable, Wi-Fi and Bluetooth. Digital images taken by the digital camera are transmitted to the PC, and the digital images are processed by using analysis software such as MATLAB. Both the digital camera and the PC are for consumer use, so are not equipped with any special function . This system will be able to measure the diameter of a running rope by using a high-speed video camera instead of the digital still camera.

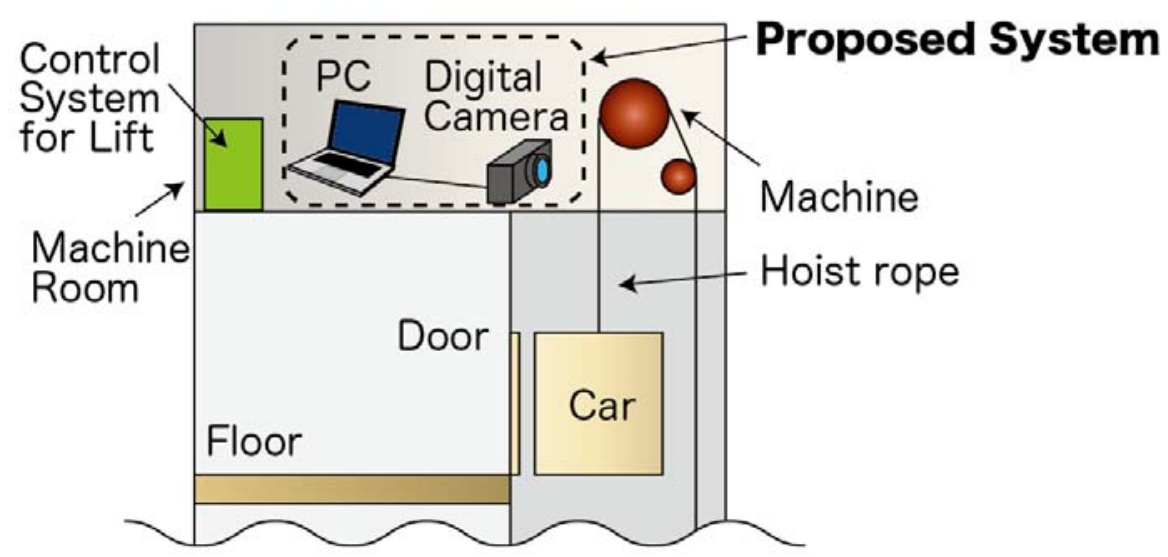

Figure 1 Concept diagram of health monitoring system 


\section{METHODOLOGY}

\subsection{Diameter measurement by image processing}

This section describes an algorithm of the diameter measurement of a rope by using image processing. Figure 2 shows a flowchart of the diameter measurement programme, and Fig. 3 is an example of image processing. The algorithm is as follows;

First of all, a digital image taken by a digital camera is loaded to a computer as shown in Fig. 3 (a).

Next any unnecessary part of the image is trimmed, and the image is changed from colour to gray scale as shown in Fig. 3 (b). The gray scale image was obtained by using the following equation;

$$
L_{G S i j}=\frac{L_{R i j}+L_{G i j}+L_{B i j}}{3}
$$

Where, $L_{G S i j}$ is a luminance of a pixel $(i, j)$ in the gray scale image, $L_{R i j}$ is a that of a red layer in the colour image, $L_{G i j}$ is a that of a green layer and $L_{B i j}$ is a that of a blue layer. Generally the colour image consists of three layered two-dimensional arrays that are the red layer, the green layer and the blue layer, and each colour is expressed by 8bit $(=256)$ data. The gray scale image has only one layer that expresses luminance, and the luminance is also expressed by 8bit $(=256)$ data. Therefore capacity of data of the image is decreased by this process.

Then a low-pass filter is applied as shown in Fig. 3 (c). This process removes noise, dust and the unevenness of the rope surface, so boundary line will be decreased. In our method, the low-pass filter using the Fourier transform was applied.

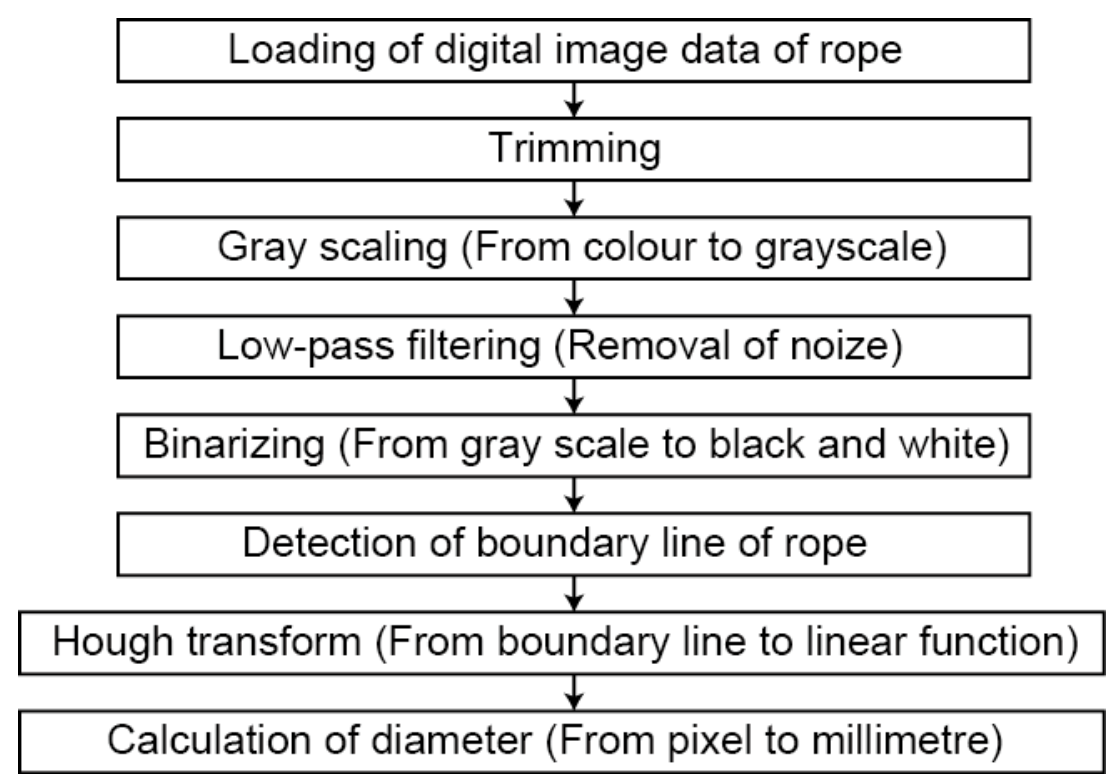

Figure 2 Flowchart of diameter measurement programme 


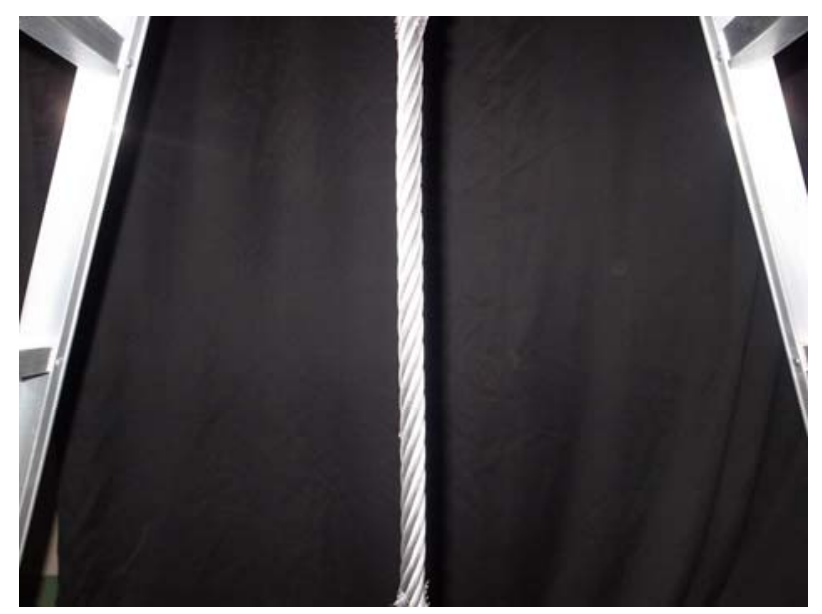

(a) Original image

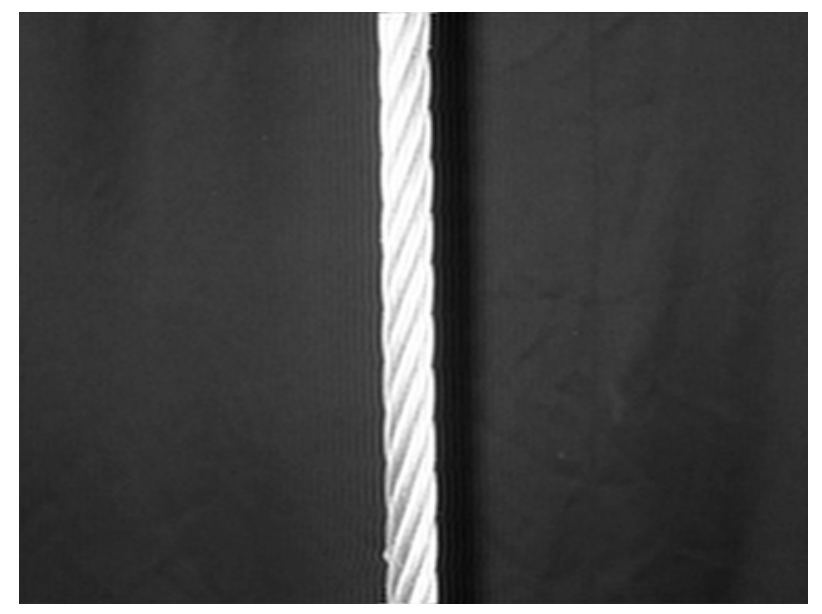

(c) Filtered image

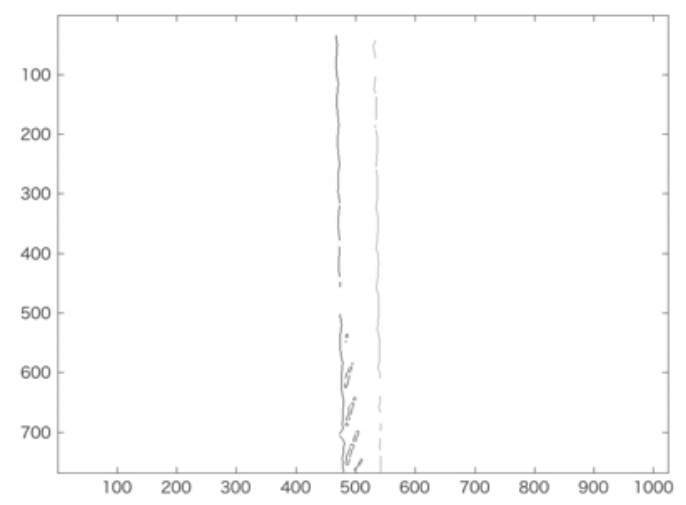

(e) Boundary line image

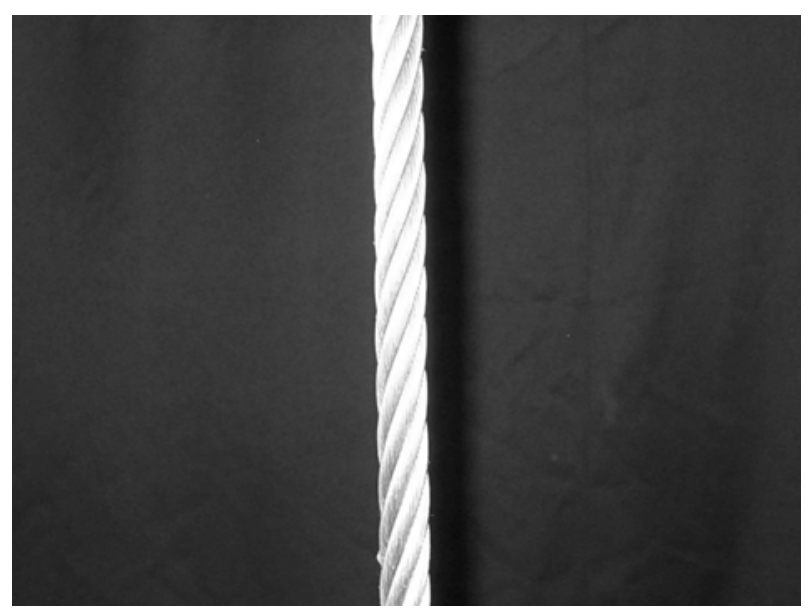

(b) Trimmed and gray scaled image
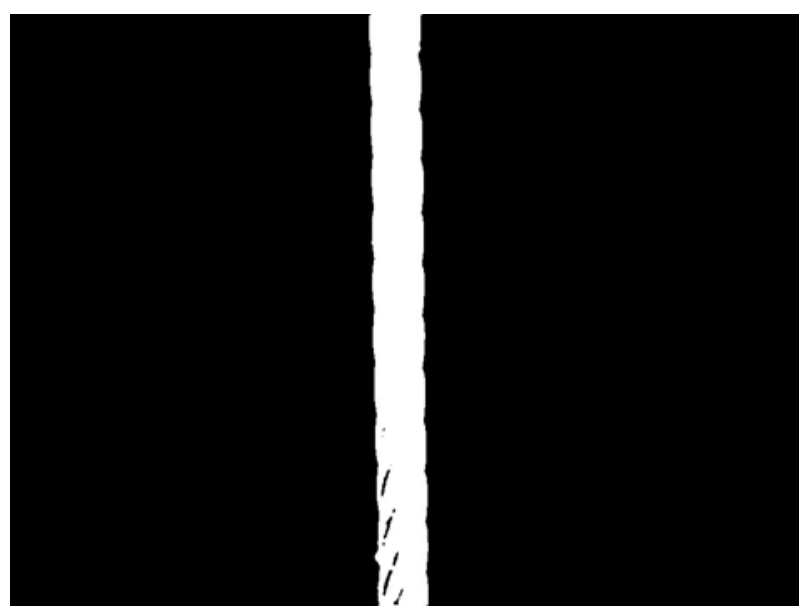

(b) Binarized image

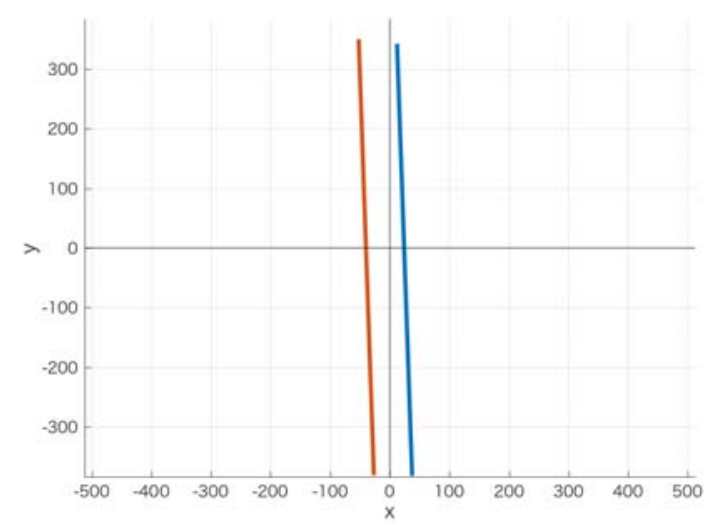

(f) Linearized image

Figure 3 Example of process of image processing 
After that, the image is changed from gray scale to monochrome as shown in Fig. 3 (d). The monochrome image was obtained by using following equation;

$$
L_{M C i j}=\left\{\begin{array}{cc}
0 & \left(L_{G S i j} \leq T_{\text {binarize }}\right) \\
1 & \left(L_{G S i j}>T_{\text {binarize }}\right)
\end{array}\right.
$$

Where, $L_{M C i j}$ is a value of a pixel $(i, j)$ in the monochrome image, $T_{\text {binarize }}$ is a threshold value that separates gray scale data into black and white. The monochrome image is expressed by 1 bit $(=2)$ data. In other words, black is expressed by 0 , and white is expressed by 1 . The threshold value should be adjusted in consideration of photographing conditions.

Then, boundary lines between white and black are detected as shown in Fig. 3 (e). In this process, the value of each pixel is checked, and the boundary is judged by comparing it with the surrounding pixels. These lines express an outline of the rope.

After that, the Hough transform [3] is applied as shown in Fig. 3 (f). Hough transform is a method that detects linear functions from a digital image. In general, a linear function is expressed by the following equation.

$$
y=\beta_{1}+\beta_{2} x
$$

However, Eq. 3 is not adequate to represent vertical lines, so this cannot be expressed in computer programmes. Therefore the line is expressed by distance from the origin $\rho$ and an angle between the horizontal axis $\theta$ as shown in Fig. 4, that is to say, polar coordinates. In the Hough transform, candidate values of $\rho$ and $\theta$ of each pixel are calculated, and the corresponding values assigned to the variables $\rho$ and $\theta$. As shown in Fig. 4, the difference $\rho-\rho$ ' is distance between two lines.

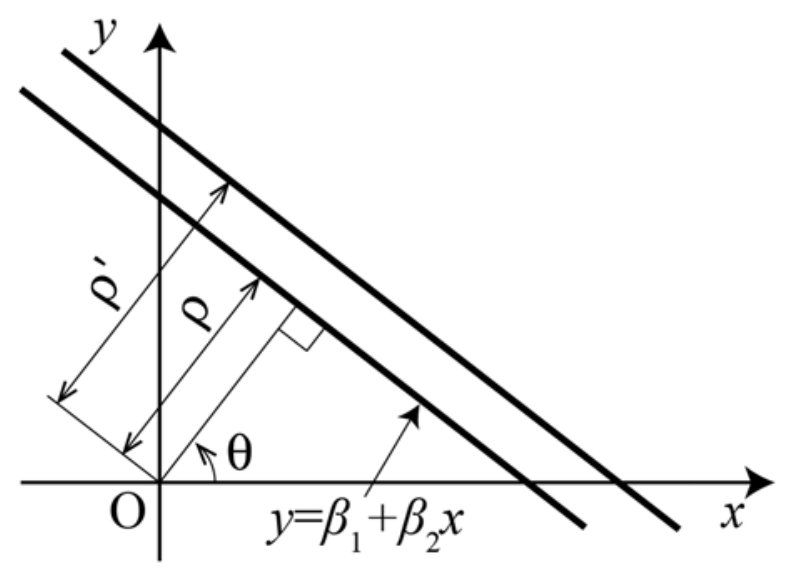

Figure 4 Linear function 
Finally, the diameter of the rope is calculated. Distance derived by the Hough transform was the distance in the digital image, so it should be transformed to length in actual scale. The pixel in the digital image is transformed to actual length by using the sensor size and a ratio of the focal length in the digital camera to the distance between the camera and rope as shown in Fig. 5.

In order to measure the diameter accurately, the distance placed between the rope and camera needs to be clear. In this paper, the distance was measured by using a scale. It is also able to measure the distance by digital images of checkerboard as shown in Fig. 6. In this method, the size of the checkers needs to be clarified. The method using checkerboard is effective in a real environment of a lift system.

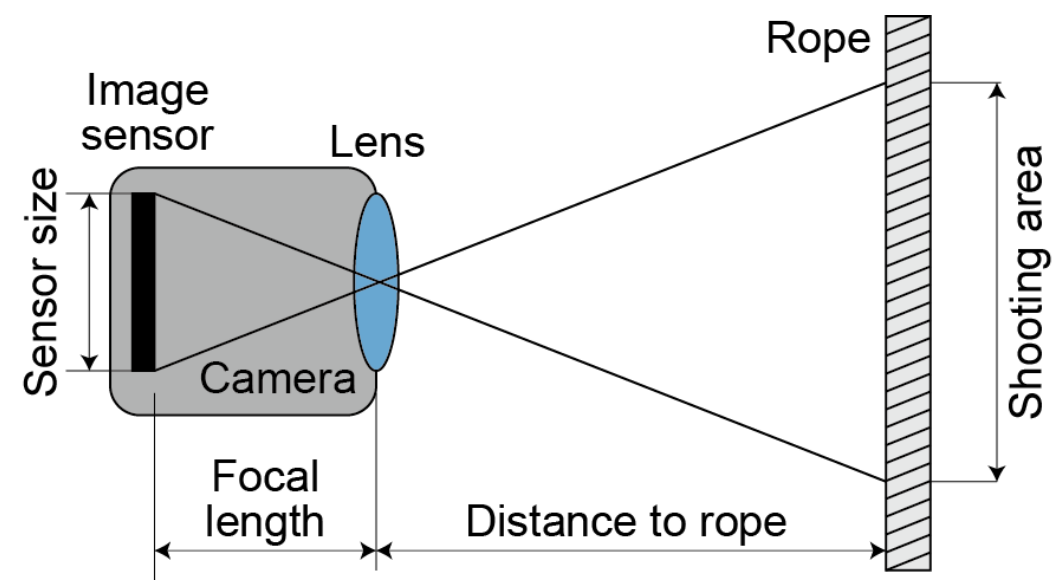

Figure 5 Calculation of actual size by specification of camera

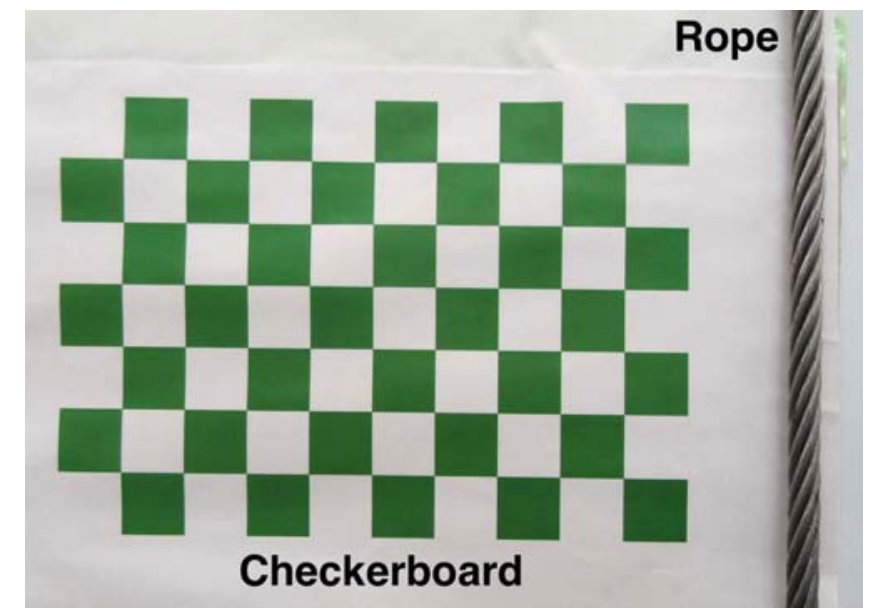

Figure 6 Checkerboard

\subsection{Red rust detection by image processing}

This section describes an algorithm of red rust detection of a rope by using image processing. Generally, a colour image consists of three layered two-dimensional arrays as shown in Fig. 7. Each 
layer indicates the luminance of red, green and blue, and each luminance is expressed by 8 bit (= 256 levels) data. Therefore a colour is identified by combination of luminance of red, green and blue.

This system detects red rust by colour recognition. First, ranges of luminance that correspond with red rust are set, that is to say, the upper and lower limits of luminance of red, green and blue are set. Next, luminance of each pixel is inspected. Finally the pixel is marked if the luminance is in the range of red rust.

Figure 8 shows an example of red rust detection, and (a) shows original image, (b) shows the red rust part. The object is a rust oilcan. The ranges of luminance of red rust were set as follows; red is 110 to 255, green and blue are 0 to 80 . Although the background of the digital image had a similar colour to red rust, red rust on the oilcan was detected well by a suitable setting of the ranges of luminance.

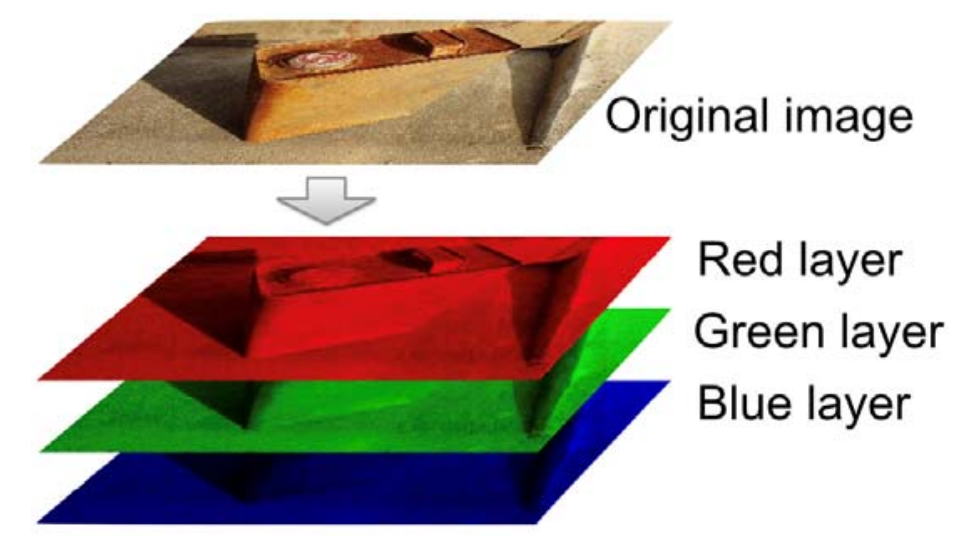

Figure 7 Resolution of digital image to RGB layer

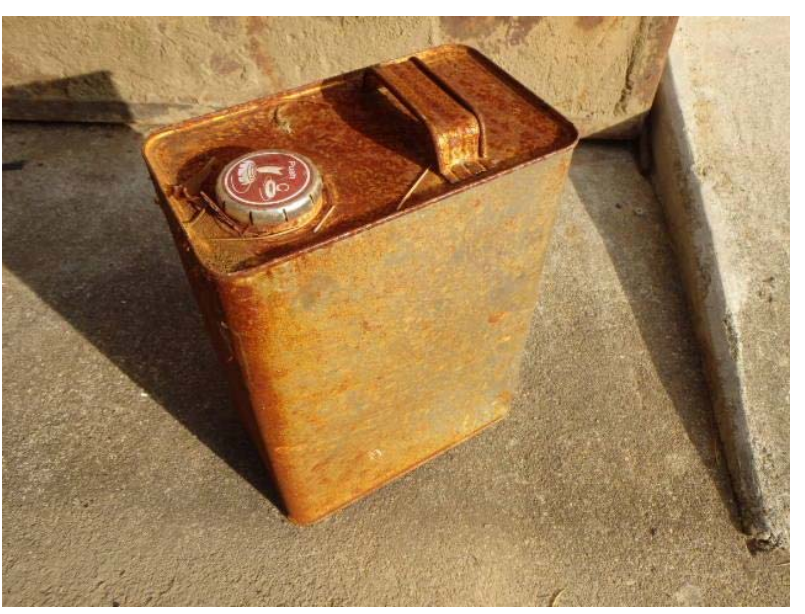

(a) Original image

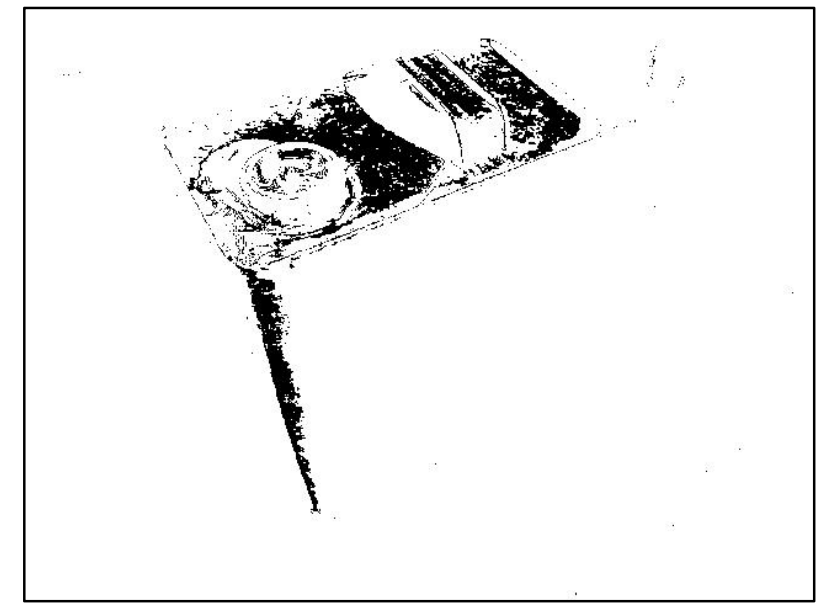

(b) Red rust part

Figure 8 Example of red rust detection 


\subsection{Outline of tests}

Verification tests were carried out in order to verify accuracy of the proposed system and to clarify suitable conditions for photography. Influence of diameter of ropes on measurement accuracy, influence of lighting conditions of photography on measurement accuracy, influence of resolution of digital images on measurement accuracy and influence of distance between wire rope and camera were investigated. In addition, the applicability of the method to the case where various ropes are in one frame was investigated in consideration of actual setups of lift systems.

Figure 9 shows an experimental apparatus. The experimental apparatus consists of a wire rope, a weight to tighten the rope, a stepladder that the rope is suspended from and a digital camera. Three wire ropes made of carbon steel were used. Diameters measured by a vernier caliper were 6.00, 9.05 and $15.95 \mathrm{~mm}$, respectively. The digital camera had a focal length of $6 \mathrm{~mm}$ and an 1/1.7-inch CCD image sensor. Distance between the rope and camera was $400 \mathrm{~mm}$.

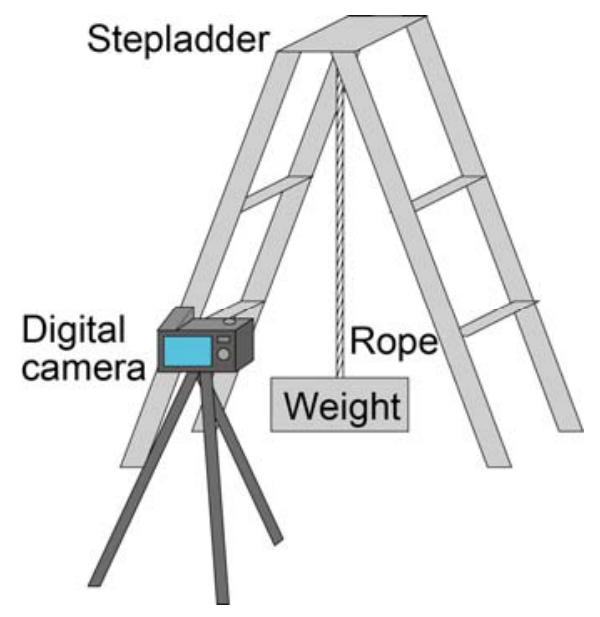

Figure 9 Experimental apparatus

\subsection{Influence of diameter of rope and room lighting condition}

The influence of the diameter of the rope and the room lighting conditions of photography were investigated. The resolution of digital images was $1280 \times 960$ pixel. In this condition, one pixel corresponded to about $0.4 \mathrm{~mm}$. Figure 10 shows the comparison of the measurement accuracy on diameters and the lighting condition. It is confirmed from Fig. 10 that the suitable lighting condition for photography is the condition without lighting and with the flash. In this condition, error ratio was less than $0.93 \%$ and there was no significant difference between diameters.

As a result, it was confirmed that the proposed system has sufficient measurement accuracy, and the condition without lighting and with the flash is required in real lift systems to obtain accuracy. 


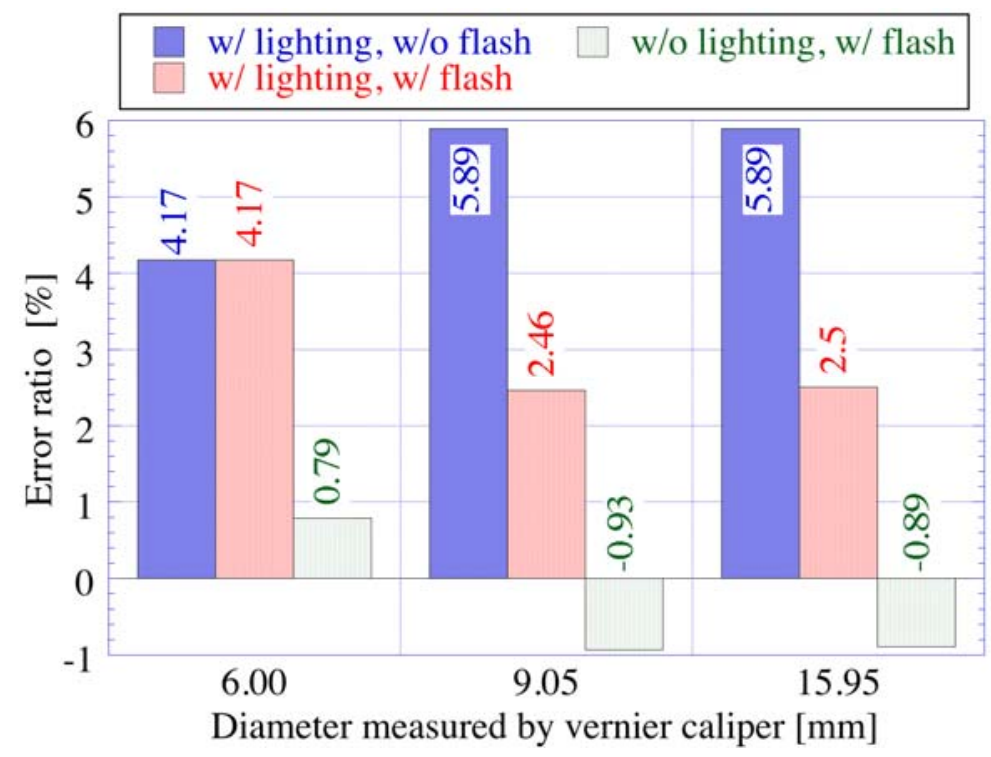

Figure 10 Influence of diameters and lighting condition

\subsection{Influence of resolution of digital image}

The influence of the resolution of digital images was investigated. A 15.95-mm-diameter wire rope was used for the test. Lighting was turned off, and the flash was turned on. Figure 11 shows comparison of measurement accuracy and processing time on resolution of digital images. The error ratio decreased by increasing resolution, because the number of pixels per mm increased. On the other hand, the processing time increased by increasing resolution, because the number of calculations increased. In addition, the error ratio of the $1280 \mathrm{x}$ 960pixel image is larger than that in Fig. 10 though the same image was used, because different parameters such as the low-pass filter and the threshold value for binarization were applied.

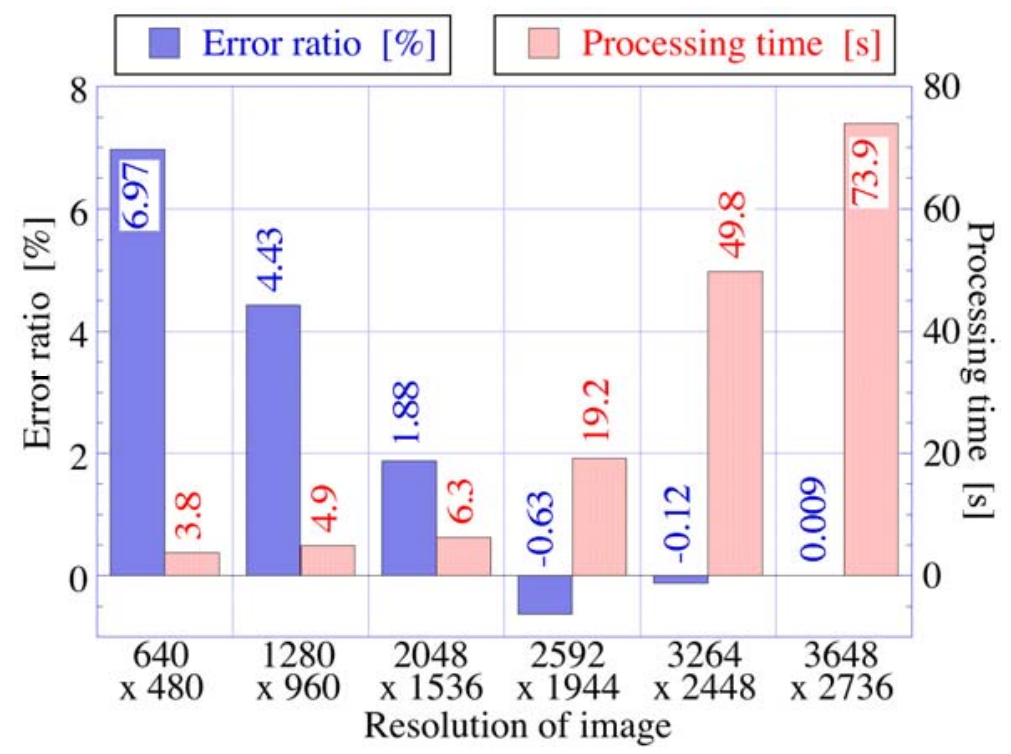

Figure 11 Influence of resolution of digital images 


\subsection{Influence of distance between wire rope and digital camera}

The influence of the distance between the wire rope and the digital camera was investigated. The distance was 300, 400 and $500 \mathrm{~mm}$. Resolution of the digital image was also changed. Three images per condition were taken, and unevenness and average of error were investigated. Figure 12 shows the relationship between the distance and the error ratio. Although there is no definite tendency between the distance and the average error ratio, the difference between the maximum and the minimum error increased when increasing the distance. Additionally, the difference was large when the resolution of the digital image was small.

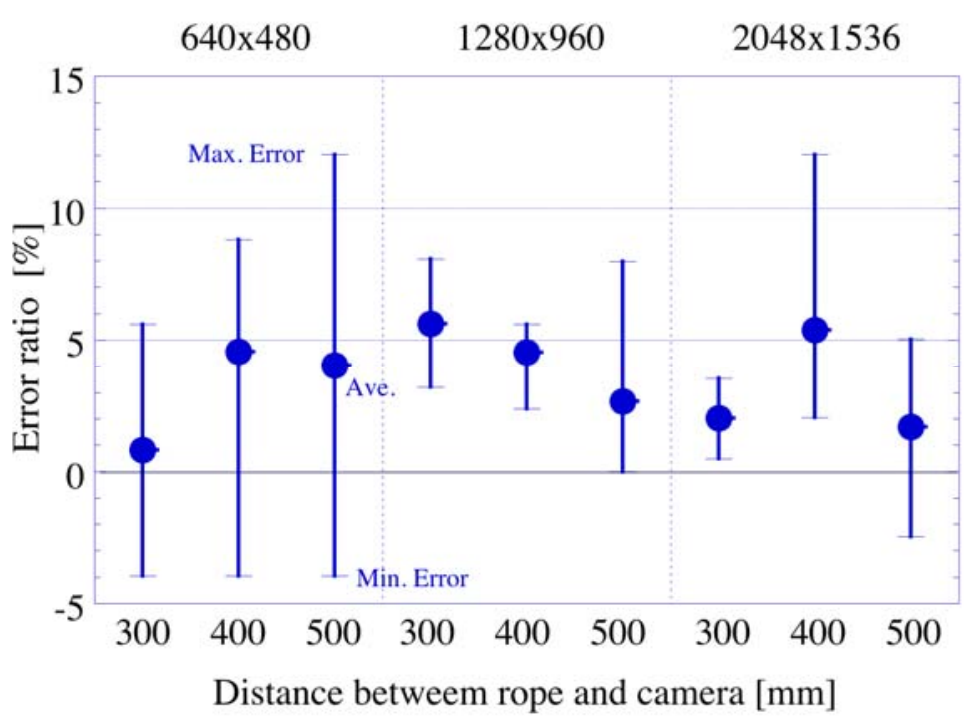

Figure 12 Influence of distance between wire rope and digital camera

\subsection{Consideration of actual setups}

In general, actual lifts are suspended by several wire ropes. For this reason, applicability of the proposed system to a digital image of a three ropes in one frame was investigated. Three ropes whose diameters were 6.00, 9.05 and $15.95 \mathrm{~mm}$ were used. Distance between the wire ropes and the digital camera was $400 \mathrm{~mm}$. Room lighting was turned off, and the flash was turned on. Resolution of digital images was 1280 x 960pixel.

Figure 13 shows the image processing results of the image of the three ropes in one frame. It was confirmed from Fig. 13 that each rope was recognized well, although the image was relatively complicated compared to the image with one rope. Figure 14 shows the error ratio of each rope. In this case, the error ratio increased with an increase of the diameter, and the maximum error ratio was less than $10 \%$. The measurement accuracy depends on parameters of image processing such as properties of the low-pass filter, threshold value for binarization and so on. Although the error ratios of the wire ropes of 6.00 and $9.05 \mathrm{~mm}$ were small, the measurement accuracy should be improved by adjusting the parameters for image processing and considering distortions of lens. 


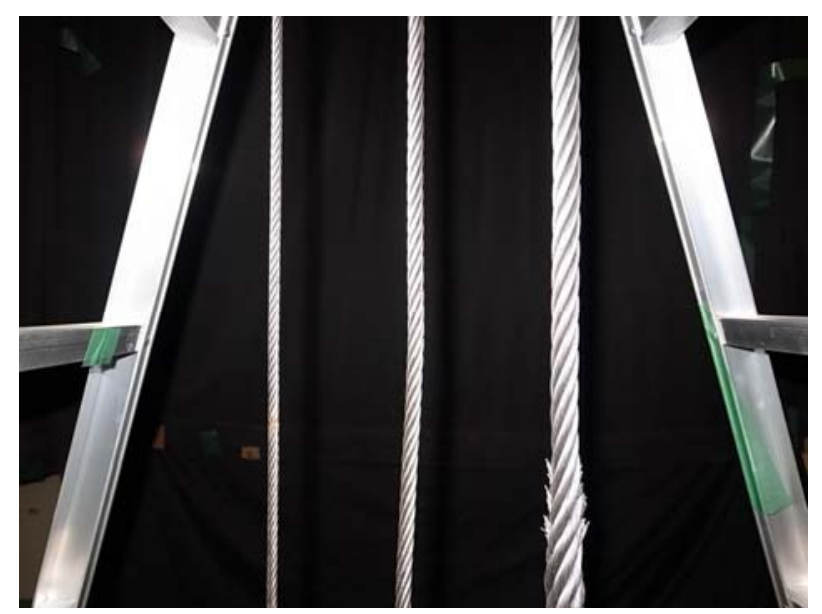

(a) Original image

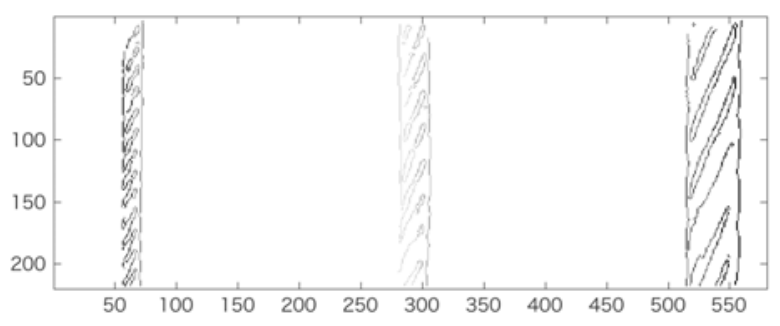

(c) Boundary line image

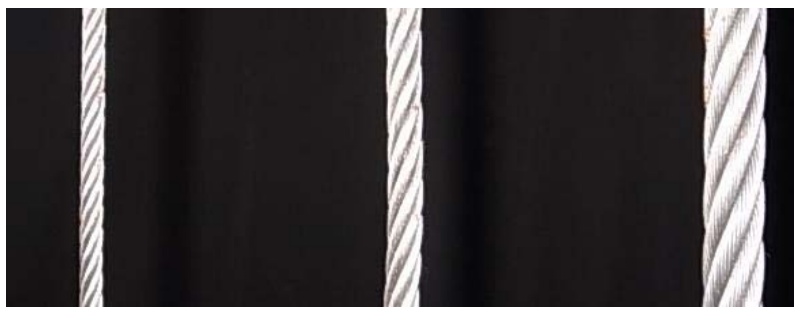

(b) Trimmed and gray scaled image

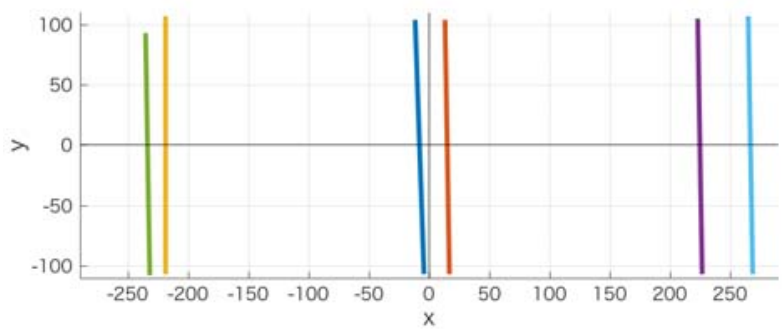

(d) Linearized image

Figure 13 Image processing results (three ropes in one image)

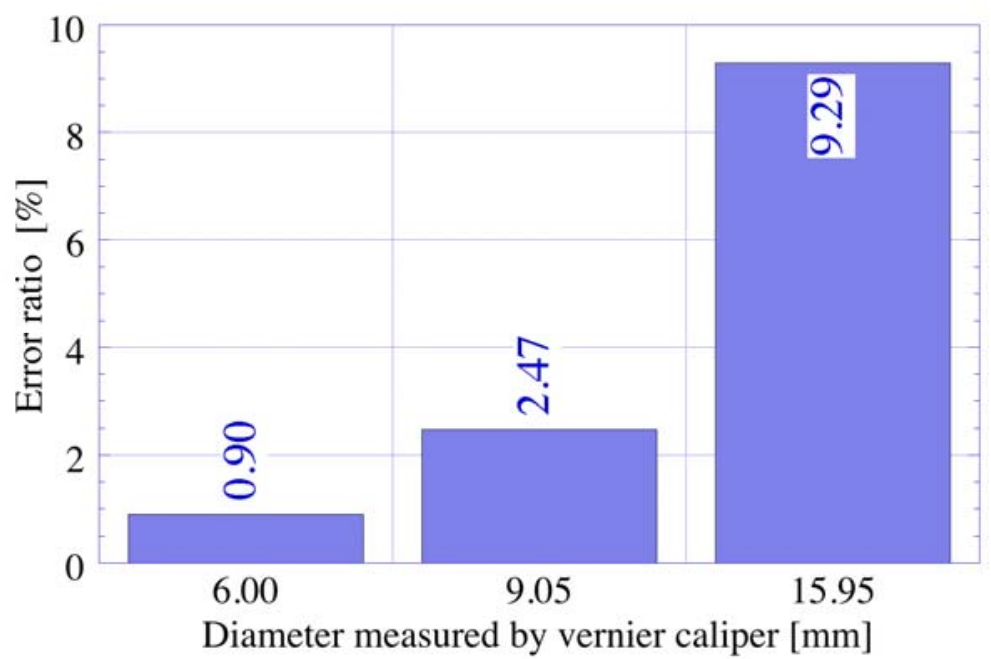

Figure 14 Error ratio of diameter measurement (three ropes in one image) 


\section{RED RUST DETECTION}

Experiments using a rust wire rope were carried out in order to verify the applicability of the red rust detection method on the proposed system. The same test apparatus as section 4 was applied in the test. The distance between the rope and the camera was $400 \mathrm{~mm}$. Resolution of digital images was 1280 x 960pixel. Lighting was turned off, and the flash was turned on.

Figure 15 shows a result of red rust detection, and (a) shows the original image, (b) shows the red rust part. The object is a rust rope made of carbon steel and having $12 \mathrm{~mm}$ diameter. The ranges of luminance of the red rust were same as in section 3.2, i.e. red is 110 to 255, green and blue are 0 to 80. Red rust on the rope was detected well as shown in Fig. 15 (b). Although red rust was detected well by using parameters that are set in section 3.2, more accurate detection is possible by adjusting these parameters.

The detection of red rust by the proposed system is efficient, because the detection is executed at the same time as the diameter measurement by image processing.

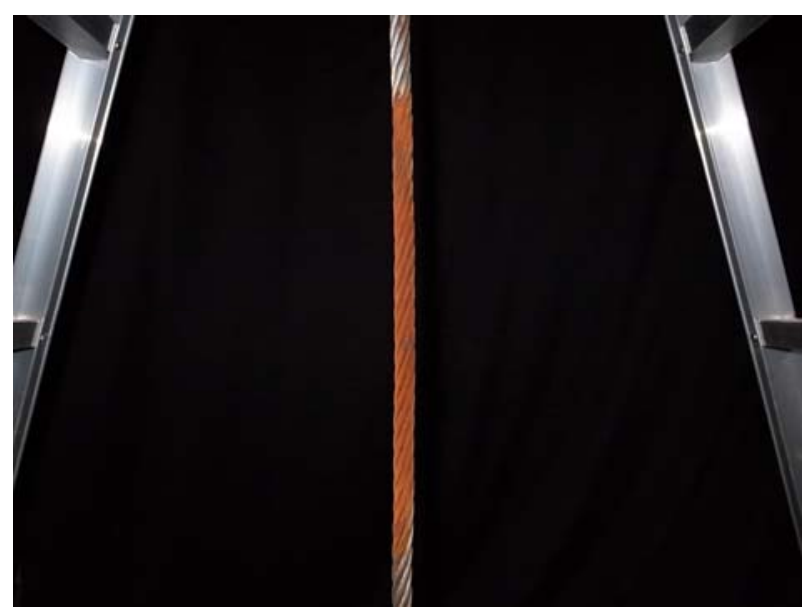

(a) Original image

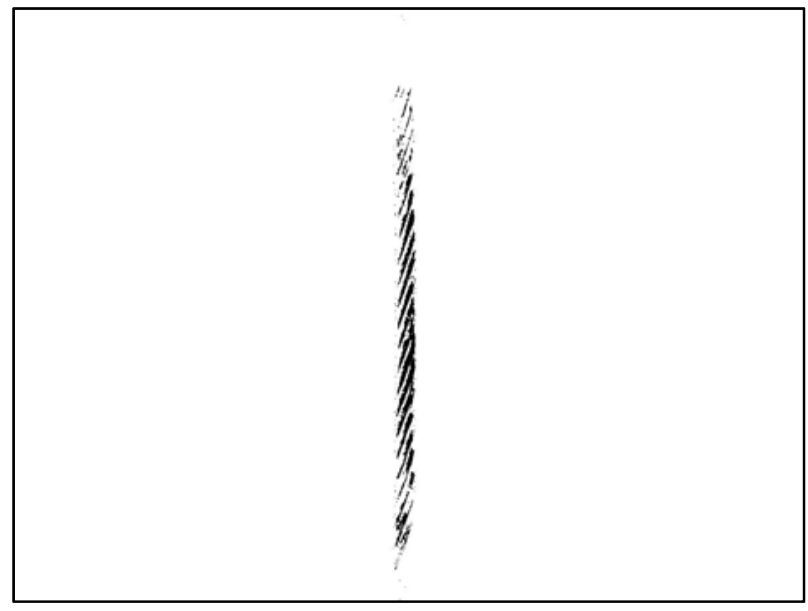

(b) Red rust part

Figure 15 Red rust detection

\section{VERIFICATION BY REAL ENVIRONMENT}

\subsection{Lift}

In order to verify applicability of the proposed system to real environment, the proposed system was applied to an actual lift.

The lift is used in the building \#27 of Saitama Institute of Technology. The maximum capacity is $600 \mathrm{~kg}$ (9 person). The lift was made in 1991, and the hoist ropes were replaced in 2007. The lift has 4 hoist ropes, and the diameters measured by a vernier caliper were 12.04, 12.08, 12.06 and 12.05mm ( $\mathrm{L}$ to R), respectively. Figure 16 shows the machine room of the lift and setup of the digital camera. 


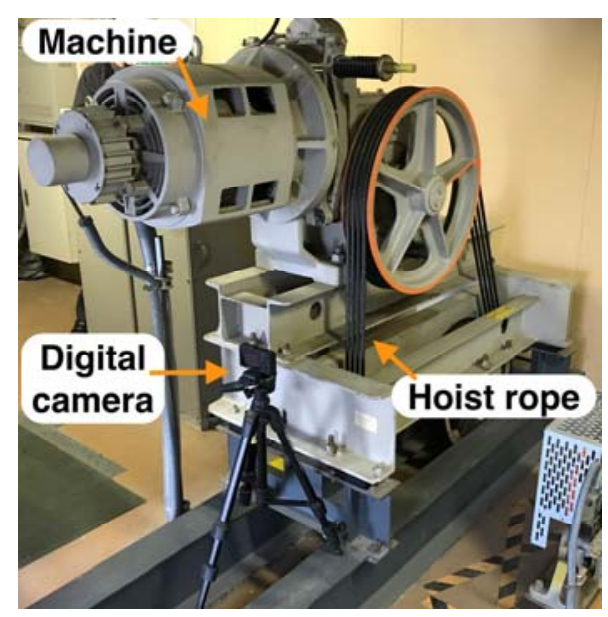

Figure 16 Verification by actual environment

\subsection{Diameter measurement}

The diameters of the ropes were measured by using the proposed system. The distance between the wire ropes and the digital camera was 300, 400 and 500mm. Room lighting was turned off, and the flash was turned on. Resolution of digital images was 2048 x 1536pixel.

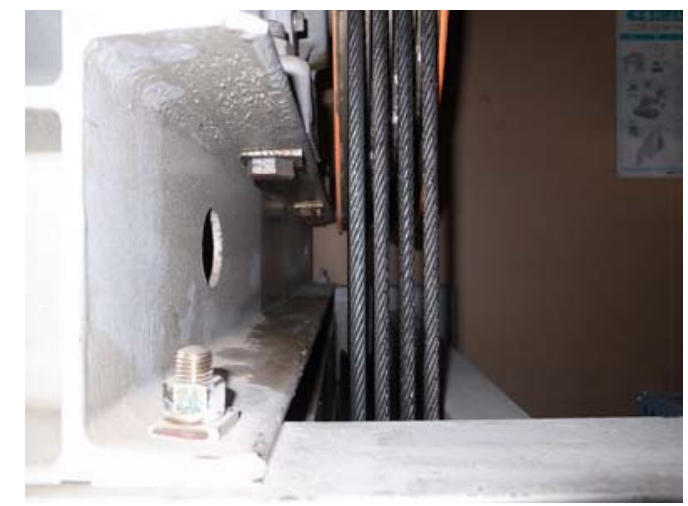

(a) Original image

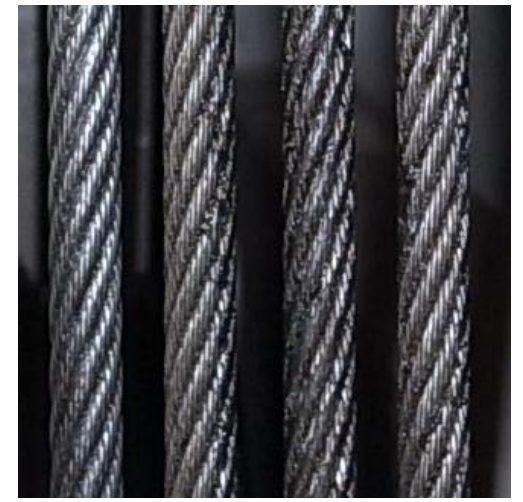

(b) Trimmed and gray scaled image

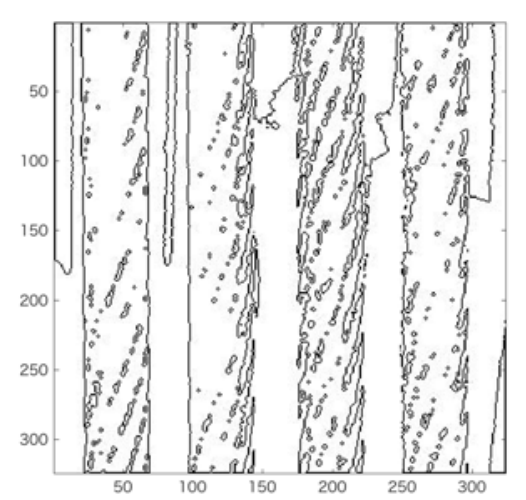

(c) Boundary line image

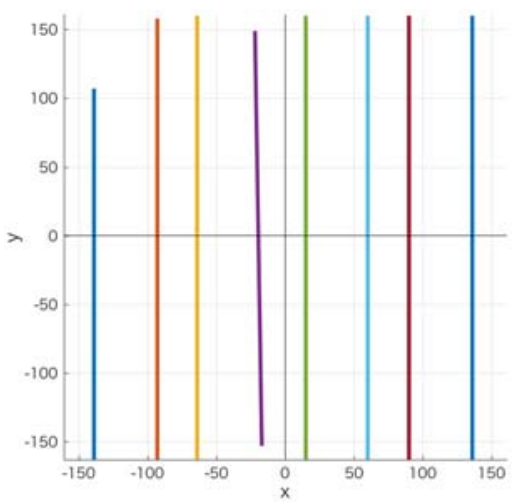

(d) Linearized image

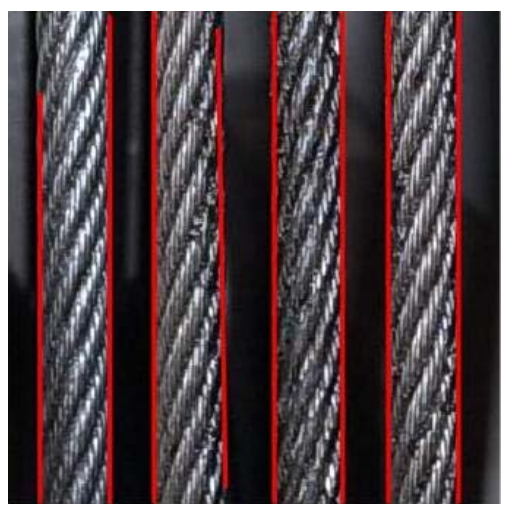

(e) Detected line on image

Figure 17 Image processing results (actual hoist ropes) 


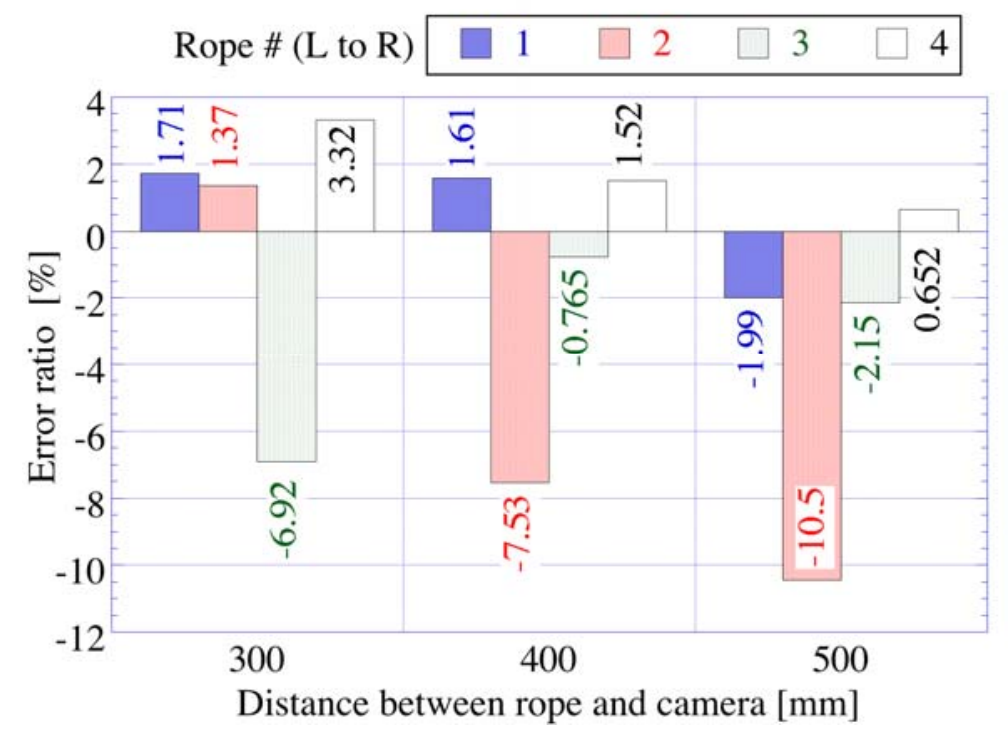

Figure 18 Error ratio of diameter by distance between rope and camera

(actual hoist ropes)

Figure 17 shows the image processing results of the actual hoist ropes. The distance between the ropes and the camera was $400 \mathrm{~mm}$. The actual ropes were recognized well as shown in Fig. 17 (e). Figure 18 shows the error ratio of each rope by the distance between the ropes and the camera. The error ratio increased with an increase of the distance. The reason is that the background is also in focus, when the distance is long. Although some results have unacceptable error, most results have good accuracy. The measurement accuracy will be improved by adjusting the photography conditions and parameters for image processing

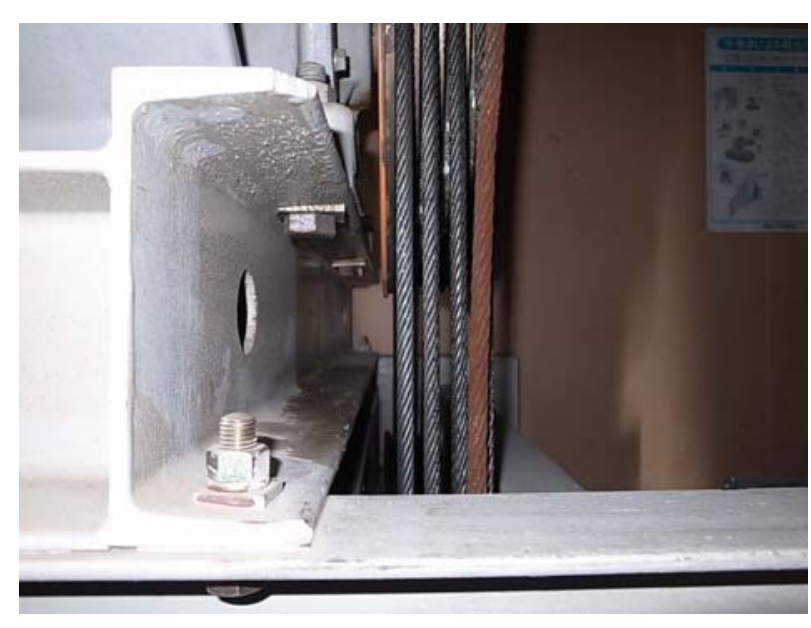

(a) Original image

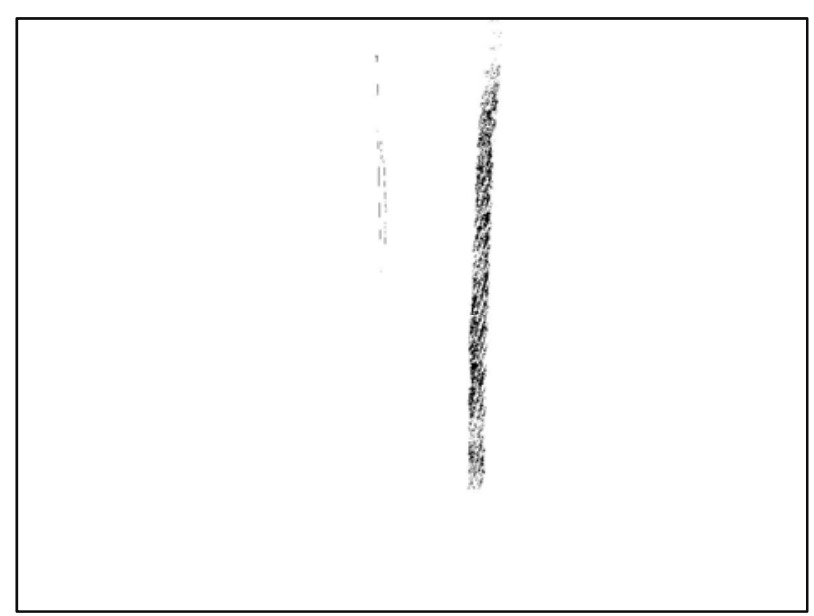

(b) Red rust part

Figure 19 Red rust detection (actual environment) 


\subsection{Red rust detection}

The proposed method for the red rust detection was verified in the actual environment. An artificially rusted wire rope of $12 \mathrm{~mm}$ in diameter was set in front of the actual hoist ropes as shown in Fig. 19 (a). The distance between the rope and the camera was $400 \mathrm{~mm}$. Resolution of digital images was 2048 x 1536pixel. Lighting was turned off, and the flash was turned on.

Figure 19 (b) shows the red rust part. The ranges of luminance of the red rust were the same as in section 3.2 and 5, i.e. red is 110 to 255, green and blue are 0 to 80 . Red rust on the rope was detected well as shown in Fig. 19 (b). Although the tangerine-coloured sheave was also detected, the area was small, so the rope parts can be distinguished. In addition, the rope parts can be distinguished by the process of the diameter measurement as well, so the detection accuracy will be improved by combining the results of diameter measurement and red rust detection.

\section{CONCLUSIONS}

This paper focused on the decrease of the diameter and occurrence of red rust on wire ropes, that is related to their rupture, and proposed the application of image processing to monitor the health of the wire rope of lift systems. The system was built using MATLAB and the accuracy was verified by various tests.

As a result, the proposed system had sufficient accuracy of diameter measurement for images with one rope regardless of diameters, and the photography condition without lighting and with flash was suitable. Although ropes in the image with three ropes were recognized well, however the measurement accuracy was insufficient. In addition, the proposed system was able to detect red rust on the wire ropes. Finally, the verification using the actual lift system was conducted. Although some results have error, most results have good accuracy, and red rust was detected well by the proposed system.

In order to improve accuracy and applicability of this system, adjustments of parameters of image processing, consideration of distortions of lens, consideration of various situations such as a rope covered with grease and development of fast algorithms are required in the future. In addition, detection of wire breakage and counting the number of breakages by image processing will also be implemented. After this method using a still camera is constructed, a high-speed video camera will be used in order to measure diameter of a running rope.

\section{REFERENCES}

[1] https://www.mlit.go.jp/common/000188107.pdf (Accessed 31 October 2017, in Japanese)

[2] Tokyo Denki University, Report on investigation regarding safety measures of hoist rope of lift systems. (2013). (in Japanese)

[3] S. Fujita, O. Furuya, Y. Niitsu, T. Mikoshiba, and D. Nakano, Research and Development of 3-D Measurement Method for Dynamic Displacement in Shake Table Tests Applying Image Processing Technique. In: Proceedings of 13th World Conference on Earthquake Engineering, Vancouver, British Columbia, Canada, 1-6 August 2004, paper \#417

[4] K. Minagawa, and S. Fujita, Health Monitoring System for Wire Rope Using Image Processing. In: Proceedings of the 7th Symposium on Lift and Escalator Technologies, Northampton, UK, 20-21 September 2017, 15-1 15-10 
[5] N. Takai, Getting Started With MATLAB Image Processing. Kogakusha, Tokyo (2013). (in Japanese) 